\title{
Use of MASCC score in the inpatient management of febrile neutropenia: a single-center retrospective study
}

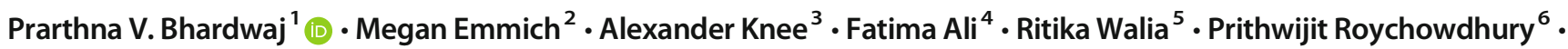 \\ Jackson Clark ${ }^{4}$ Arthi Sridhar ${ }^{7} \cdot$ Tara Lagu $^{8,9} \cdot$ Kah Poh Loh $^{10}$
}

Received: 9 January 2021 / Accepted: 15 March 2021 / Published online: 24 March 2021

(C) The Author(s), under exclusive licence to Springer-Verlag GmbH Germany, part of Springer Nature 2021

\begin{abstract}
Purpose The Multinational Association for Supportive Care in Cancer (MASCC) score is used to risk stratify outpatients with febrile neutropenia (FN). However, it is rarely used in hospital settings. We aimed to describe management, use of MASCC score, and outcomes among hospitalized patients with FN.

Methods We conducted a retrospective cohort study of patients with cancer and FN. We collected patient demographics, cancer characteristics, microbiological profile, MASCC score, utilization of critical care therapies, documentation of goals of care (GOC), and inpatient deaths. Outcomes associated with low- $(\geq 21)$ versus high-risk $(<21)$ MASCC scores are presented as absolute differences. Results Of 193 patients, few $(2 \%, n=3)$ had MASCC scores documented, but when calculated, $52 \%(n=101)$ had a high-risk score $(<21)$. GOC were discussed in $12 \%(n=24)$ of patients. Twenty one percent $(n=40)$ required intermediate/ICU level of care, and $12 \%(n=23)$ died in the hospital. Those with a low-risk score were $33 \%$ less likely to require intermediate/ICU care (95\% CI 23 to 44\%) and 19\% less likely to die in the hospital (95\% CI 10\% to 27\%) compared to those with high-risk score.

Conclusions MASCC score was rarely used for hospitalized patients with FN, but high-risk score was associated with worse outcomes. Education efforts to incorporate MASCC score into the workflow may help identify patients at high risk for complications and help clinicians admit these patients to a higher level of care (e.g., intermediate/ICU care) or guide them to initiate earlier GOC discussions.
\end{abstract}

Keywords Febrile neutropenia $\cdot$ Cancer $\cdot$ Goals of care $\cdot$ Multinational Association for Supportive Care Score $\cdot$ Neutropenic fever

\section{Introduction}

Febrile neutropenia (FN) is a common complication in patients with cancer, occurring in 5-10\% of patients with solid

Dr. Lagu and Dr. Loh are shared senior authors.

Prarthna V. Bhardwaj

prarthna.bhardwaj@baystatehealth.org

Megan Emmich

Megan.emmich@gmail.com

Alexander Knee

Alexander.knee@baystatehealth.org

Fatima Ali

Fatima.alimd@baystatehealth.org

Ritika Walia

Ritika.waliamd@baystatehealth.org tumors [1], 85-95\% of patients with acute leukemia, and 20$25 \%$ of patients with other hematologic malignancies [2]. Neutropenia is defined as an absolute neutrophil count (ANC) of $<500$ neutrophils $/ \mathrm{mcL}$ or the combination of

\author{
Prithwijit Roychowdhury \\ Prithwijit.roychowdhury@umassmed.edu \\ Jackson Clark \\ Jackson.clarkdo@baystatehealth.org \\ Arthi Sridhar \\ artshri@gmail.com \\ Tara Lagu \\ lagutc@gmail.com \\ Kah Poh Loh \\ kahpoh_loh@urmc.rochester.edu
}

Extended author information available on the last page of the article 
ANC of $<1000$ neutrophils/mcL and a predicted decline of $\leq$ 500 neutrophils $/ \mathrm{mcL}$ over the next $48 \mathrm{~h}$. FN exists once there is a temperature $\geq 38.3{ }^{\circ} \mathrm{C}$ orally or $\geq 38{ }^{\circ} \mathrm{C}$ for a duration longer than $1 \mathrm{~h}$ with associated neutropenia [3]. FN accounts for about $40-50 \%$ of the total cost of hospitalizations in cancer care, with mortality rates ranging between 3 and $10 \%$ [4].

Prior literature reports that $20-30 \%$ of patients with FN have an identifiable site of infection and $10-25 \%$ of patients have positive blood cultures [5-8]. Experts recommend the early introduction of empiric antibiotic therapy in all patients with FN because of the high risk for progression to sepsis and death $[7,9,10]$. Historically, Pseudomonas aeruginosa was the most prevalent causative organism, but the changing microbiological profile of FN now reveals that gram-positive bacteria are more common [11, 12].

As patients at low risk for complications could be successfully treated in the outpatient setting, several organizations including the American Society of Clinical Oncology (ASCO), National Comprehensive Cancer Network (NCCN), Infectious Disease Society of America (IDSA), and European Society of Medical Oncology (ESMO) recommend using the Multinational Association for Supportive Care in Cancer (MASCC) score to risk stratify patients [7, 13, 14]. Developed in 2000, the score incorporates patient and cancer characteristics to quantify the risk of FN-related complications. Higher scores indicate lower risk, with a maximum of 26 points. A cutoff value of $\geq 21$ points is used to discriminate patients with low risk from those with high risk $(<21$ points) for serious FN-related complications [15]. Patients identified as low risk may be managed in the outpatient setting safely with oral antibiotics. Several studies have validated this score for both hematological and solid tumors in the inpatient setting and recommend early discharge of patients with low-risk FN on oral antibiotics [16-19]. Despite evidence supporting its use in the inpatient setting, MASCC score is rarely used during hospitalization $[20,21]$.

FN admissions are commonly managed by hospitalists or internists rather than oncologists in the community; a tool like the MASCC score could be used to guide them around risk for clinical deterioration or death [22]. Patients with FN and lower MASCC score might be placed in a higher level of care or, alternatively, be prioritized for earlier discussion around goals of care. Therefore, we aimed to describe the management of FN among hospitalized patients with cancer, current use of MASCC score, and the association of MASCC score on presentation with poor outcomes.

\section{Methods}

Design, setting, and population We conducted a retrospective cohort study at a tertiary care academic medical center. The Institutional Review Board (IRB) at Baystate Medical Center approved this study. We identified patients hospitalized with a principal diagnosis of FN and cancer admitted to Baystate Medical Center between October 1, 2015, and April 1, 2019. Patients were included in the study if they were 18 years of age or older, were admitted to the inpatient service or intensive care unit (ICU), and carried both a diagnosis of cancer and FN. FN was identified using ICD-10 diagnosis codes (D70 and R50) in an administrative billing database. We also included patients with cancer who were admitted for an alternative diagnosis if they developed FN during their hospitalization. If patients developed multiple episodes of FN, we randomly selected a single admission for this study. We excluded patients who did not meet diagnostic criteria for FN (defined below) and those transferred out of our facility to another acute care hospital. FN related to causes other than cancer (e.g., primary immunodeficiency or liver disease) was also excluded from the study.

Data collection Two physicians (PB and ME) reviewed the electronic health record (EHR), first examining approximately 10 of the same patients' records. Any differences were resolved via discussion to standardize chart extraction and reduce inter-observer variability. Four other physicians (FA, JC, AS, and RW) who conducted chart extraction were subsequently trained by $\mathrm{PB}$ and $\mathrm{ME}$ to ensure uniformity in data collection. After collection of data, PB and ME randomly selected 75 charts for quality control purposes and confirmed uniformity of extraction methods.

Variables extracted from the EHR included patient demographics; Eastern Cooperative Oncology Group (ECOG) performance status; use of granulocyte colony-stimulating factor (GCSF) 14 days prior to or during hospitalization; documentation of MASCC score; clinical characteristics of cancer including type, stage, and cycle of chemotherapy received; microbiological profile including the presence of positive blood cultures, source of infection, initial empiric antibiotics, use of antimicrobial prophylaxis, duration of antibiotics, and documentation of goals of care discussion; and utilization of different consult services including oncology, infectious diseases, and palliative care. Clinical course including ICU level of care, septic shock requiring vasopressor use, acute respiratory failure requiring non-invasive or mechanical ventilation, documented delirium, acute kidney injury requiring hemodialysis, and inpatient death was also obtained. Admission to the ICU and the need for critical care therapies were obtained from a review of medical records. In-hospital mortality was identified by documentation of discharge status as "expired" in our billing database. All data were manually entered into the REDCap database [23].

Definition of febrile neutropenia We defined FN based on criteria from the IDSA as an ANC of $<500$ neutrophils $/ \mathrm{mcL}$ or an ANC of $<1000$ neutrophils $/ \mathrm{mcL}$ and a predicted decline 
of $\leq 500$ neutrophils $/ \mathrm{mcL}$ over the next $48 \mathrm{~h}$ as well as a temperature of $\geq 38.3$ or $\geq 38{ }^{\circ} \mathrm{C}$ for longer than $1 \mathrm{~h}$ either prior to admission or during hospitalization when admitted for an alternative diagnosis [7].

ECOG performance status ECOG performance status describes a patient's level of functioning in terms of their ability to care for themselves, daily activity, and physical ability [24]. It ranges from 0 to 5 with 0 indicating a fully active person who is able to carry on all pre-disease performance without restriction and 5 indicating death. This score was obtained as documented on the last oncology outpatient note prior to hospitalization or oncology consult note during hospitalization.

MASCC scoring system MASCC scores were calculated by study investigators for all patients irrespective of documentation status. We calculated the weighted MASCC risk index using the following variables: burden of illness, blood pressure, presence or absence of chronic obstructive pulmonary disease, solid tumor or hematological malignancy with or without a history of previous fungal infection, dehydration, inpatient or outpatient status at the time of onset of neutropenic fever, and age. Table 1 provides details about the calculation of the score and weighting of characteristics.

\section{Statistical analyses}

We used descriptive statistics (means, standard deviations, range, and percent as appropriate to the scale of measurement) to describe the patient sample and characteristics of those with low- versus high-risk MASCC scores. Low- versus high-risk MASCC scores were used to predict hospital outcomes such as the presence of positive blood cultures, goals of care discussions, requirement of intermediate/ICU care, and death. For each association, we estimated the area under the curve (AUC) and calculated the absolute risk difference and 95\% confidence interval $(\mathrm{CI})$. The analysis was conducted using Stata v16.0, StataCorp, LP, College Station, TX.

\section{Results}

\section{Patient characteristics}

Of the 322 unique patients identified as having FN between October 1, 2015, and April 1, 2019, we excluded 129 patients; 100 patients did not meet the criteria for FN, 23 patients did not have a diagnosis of cancer, 5 patients did not meet criteria for FN or have a diagnosis of cancer, and 1 patient was transferred out of our facility. Of the 193 eligible patients, the mean age was 60 years, and more than half $(107 / 193,55.4 \%)$ were women. Most patients were white $(162 / 193,83.9 \%)$. ECOG performance status was documented for $63.7 \%$ (123/193) patients. Of these, $74.0 \%(91 / 123)$ had an ECOG of 0 or 1, and $7.3 \%(9 / 123)$ had an ECOG of 3 or 4 (Table 2).

Nearly half the patients $(102 / 193,52.5 \%)$ had hematological malignancies, with most of these being acute myeloid leukemia (33/102, 32.4\%), and non-Hodgkin lymphoma (28/ $102,27.5 \%)$. Among solid tumors, breast $(31 / 91,34.1 \%)$, thoracic $(21 / 91,23.1 \%)$, and gastrointestinal $(14 / 91,15.4 \%)$ were the most common cancers. Over two-thirds of patients with solid tumors $(64 / 91,70.3 \%)$ were clinical stage III or IV.

Most patients were receiving chemotherapy (172/193, $89.1 \%$ ) at the time of diagnosis of FN. The median cycle of chemotherapy patients received was one (range, 1-4) (Table 2). More than a third of the patients $(66 / 193,34.2 \%)$ had used GCSF 14 days prior to hospitalization or were given GCSF during hospitalization.

A MASCC score was documented in 1.6\% (3/193) of all patients by the admitting provider. A MASCC score was calculated for all patients by the study investigators based on information available from admission and emergency department visit note (Table 1). More than half of the patients $(101 / 193,52.3 \%)$ were high risk based on MASCC score of $<21$, and the remainder $(92 / 193,47.7 \%)$ were low risk. Of the patients with hematological malignancies, $57.8 \%(59 / 102)$ were considered high risk, and $42.2 \%(43 / 102)$ were considered low risk. Of the patients with solid tumors, $46.2 \%$ (42/ 91) were considered high risk, and $53.8 \%$ (49/91) were considered low risk.

\section{Microbiological profile (Table 2)}

A definitive source of infection was identified in $59.1 \%$ (114/ $193)$ of patients. A quarter of the patients $(53 / 193,27.5 \%)$ had positive blood cultures, with a slight preponderance of gramnegative rods $(26 / 53,49.1 \%)$ when compared to grampositive cocci $(23 / 53,43.4 \%)$. Forty percent $(41 / 101)$ of patients with high-risk MASSC score had positive cultures, while $13.0 \%$ (12/92) of patients with low-risk MASSC score had positive cultures.

Resistant organisms such as methicillin-resistant Staphylococcus aureus (MRSA) or pseudomonas were relatively rare, occurring in only 5.6\% (3/53) and 7.5\% (4/53) of patients, respectively. Forty-two percent $(81 / 193)$ received empiric antibiotics for MRSA coverage, and 96.4\% (189/ 193 ) received empiric antibiotics for pseudomonas coverage (Table 3).

A viral infection was identified in $15.5 \%(30 / 193)$ of patients. Approximately $11.9 \%$ (12/101) of patients with high-risk MASCC score and $19.6 \%$ (18/92) of patients with low-risk MASCC score had a viral infection. 
Table 1 Multinational Association for Supportive Care in Cancer scoring system [37]

\begin{tabular}{|c|c|c|c|}
\hline Calculated MASCC score & $\begin{array}{l}\text { Total } \\
N=193\end{array}$ & $\begin{array}{l}<21 \text { (high risk) } \\
N=101\end{array}$ & $\begin{array}{l}\geq 21 \text { (low risk) } \\
N=92\end{array}$ \\
\hline Mean (SD) & $18.51(4.99)$ & $14.86(4.15)$ & $22.52(1.61)$ \\
\hline Median (Q1, Q3) & $20.0(16.0,22.0)$ & $16.0(11.0,19.0)$ & $22.5(21.0,23.5)$ \\
\hline Min, Max & $4.0,26.0$ & $4.0,20.0$ & $21.0,26.0$ \\
\hline \multicolumn{4}{|l|}{ MASCC components } \\
\hline \multicolumn{4}{|l|}{ Burden of illness } \\
\hline Severe $(0)$ & $37(19.2 \%)$ & $37(36.6 \%)$ & $0(0.0 \%)$ \\
\hline Moderate $(+3)$ & $88(45.6 \%)$ & $56(55.4 \%)$ & $32(34.8 \%)$ \\
\hline None or mild $(+5)$ & $68(35.2 \%)$ & $8(7.9 \%)$ & $60(65.2 \%)$ \\
\hline \multicolumn{4}{|l|}{ Hypotension (SBP < 90) } \\
\hline Yes $(0)$ & $33(17.1 \%)$ & $33(32.7 \%)$ & $0(0.0 \%)$ \\
\hline No $(+5)$ & $160(82.9 \%)$ & $68(67.3 \%)$ & $92(100.0 \%)$ \\
\hline \multicolumn{4}{|l|}{ Active COPD } \\
\hline Yes $(0)$ & $15(7.8 \%)$ & $15(14.9 \%)$ & $0(0.0 \%)$ \\
\hline No $(+4)$ & $178(92.2 \%)$ & $86(85.1 \%)$ & $92(100.0 \%)$ \\
\hline \multicolumn{4}{|l|}{ Cancer type } \\
\hline Hematological malignancy with prior fungal infection $(0)$ & $22(11.4 \%)$ & $21(20.8 \%)$ & $1(1.1 \%)$ \\
\hline Solid tumor or hematological malignancy with no prior fungal infection $(+4)$ & $171(88.6 \%)$ & $80(79.2 \%)$ & $91(98.9 \%)$ \\
\hline \multicolumn{4}{|l|}{ Dehydration requiring IV fluids } \\
\hline Yes $(0)$ & $142(73.6 \%)$ & $89(88.1 \%)$ & $53(57.6 \%)$ \\
\hline No $(+3)$ & $51(26.4 \%)$ & $12(11.9 \%)$ & $39(42.4 \%)$ \\
\hline \multicolumn{4}{|l|}{ Status at onset of fever } \\
\hline Inpatient $(0)$ & $41(21.2 \%)$ & $32(31.7 \%)$ & $9(9.8 \%)$ \\
\hline Outpatient $(+3)$ & $152(78.8 \%)$ & $69(68.3 \%)$ & $83(90.2 \%)$ \\
\hline \multicolumn{4}{|l|}{ Age at onset } \\
\hline$\geq 60(0)$ & $111(57.5 \%)$ & $78(77.2 \%)$ & $33(35.9 \%)$ \\
\hline$<60(+2)$ & $82(42.5 \%)$ & $23(22.8 \%)$ & $59(64.1 \%)$ \\
\hline
\end{tabular}

Points attributed to the variable "burden of illness" are not cumulative. The maximum theoretical score is therefore 26

Table adapted from Klastersky et al.

\section{Consults (Table 2)}

Half of the patients were seen by an infectious disease specialist $(98 / 193,50.8 \%), 82.4 \%(159 / 193)$ were seen by a hematologist/oncologist, and $6.7 \%(13 / 193)$ were seen by a palliative care specialist.

\section{Goals of care discussions (Table 2)}

Goals of care discussion were initiated in 24/193 (12.4\%) of patients; the primary team/hospitalists were responsible for the majority $(19 / 24,79.2 \%)$. The oncology team initiated this conversation for 4/24 (16.7\%) patients; all of them had a highrisk MASCC score.

\section{Outcomes (Table 3)}

Inpatient death occurred in $11.9 \%(23 / 193)$ and $20.7 \%$ (40/193) required ICU level of care. About 9.8\% (19/ 193) of our sample required vasopressor support for septic shock, 9.3\% (18/193) developed respiratory failure during their hospitalization requiring either non-invasive or mechanical ventilation, $6.2 \%(12 / 193)$ had documented delirium, and $1.0 \%(2 / 193)$ had acute kidney injury requiring dialysis. Of the patients who died, 69.6\% (16/23) patients required intermediate/ICU hospitalization. Of these 16 patients, 12 of them had documented goals of care discussion. Mean MASCC score was lower among patients who died vs. those who survived (14.0 vs. 19.1) and those with positive vs. negative blood cultures (16.0 vs. 19.4).

We observed that MASCC score was predictive of poor outcomes (AUC's ranging from 0.67 to 0.76 ). In particular, those with a low-risk MASCC score were $33 \%$ less likely to require intermediate/ICU care $(95 \% \mathrm{CI}=23$ to $44 \%$ ) compared to those with a high-risk MASCC score. In addition, those with a low-risk MASCC score were $28 \%$ less likely to have a positive blood culture $(95 \% \mathrm{CI}=16$ to $39 \%)$ and nearly $19 \%$ less likely to die in the hospital $(95 \% \mathrm{CI}=10$ to $27 \%)$ compared to those with a high-risk MASCC score. 
Table 2 Baseline characteristics of patients

Patient characteristics

\begin{tabular}{l}
\hline Age, mean (SD) \\
Female, $n(\%)$ \\
Race and ethnicity, $n(\%)$ \\
White \\
Hispanic \\
African American \\
Asian/unknown
\end{tabular}
Insurance type, $n(\%)$
Medicare/Medicaid
Private

Documented ECOG PS, $n(\%)^{\alpha}$

$$
\begin{aligned}
& 0 \\
& 1 \\
& 2 \\
& 3 \\
& 4
\end{aligned}
$$$$
1
$$

Documentation of MASCC score, $n(\%)$

Cancer characteristics

Hematological malignancy, $n(\%)$

AML

Other hematologic malignancy ${ }^{n}$

Non-Hodgkin lymphoma

ALL

CLL

Hodgkin lymphoma

Multiple myeloma

CML

Solid tumors, $n(\%)$

Breast

Thoracic $^{\epsilon}$

Gastrointestinal $^{\infty}$

Genitourinary $^{£}$

Head and neck

Gynecologic ${ }^{¥}$

Soft tissue (non-lymphomas)

CNS tumors (brain, spinal cord-non-myeloma)

Stage of cancer (solid tumors only; $N=91$ )

I
II
III

IV

Patient receiving chemotherapy, Yes, $n(\%)$

Cycle of chemotherapy ( $n=172$ ), median (IQR)

Use of GCSF prior/during hospitalization, $n(\%)$

Use of prophylactic antimicrobials, Yes, $n(\%)$

Microbiological profile

Viral infection at time of admission

Positive blood cultures, $n(\%)$

Gram-positive cocci

MRSA

Gram-negative rods

Pseudomonas

Fungi $^{\beta}$
MASCC score

\begin{tabular}{ll}
\hline $\begin{array}{l}<1 \text { (high risk) } \\
N=101(52.3 \%)\end{array}$ & $\begin{array}{l}\geq 21(\text { low risk) } \\
N=92(47.7 \%)\end{array}$ \\
\hline $64.9(14.3)$ & $55.3(14.1)$ \\
$50(49.5)$ & $57(62.0)$ \\
& \\
$90(89.1)$ & $72(78.3)$ \\
$7(6.9)$ & $11(12.0)$ \\
$3(3.0)$ & $7(7.6)$ \\
$1(1.0)$ & $2(2.2)$
\end{tabular}

$116(61.1)$

68 (68.7)

$48(52.8)$

74 (38.9)

$31(31.3)$

43 (47.3)

$123(63.7)$

43 (35.0)

48 (39.0)

23 (18.7)

$8(6.5)$

$1(0.8)$

3 (1.6)

58 (57.4)

14 (24.1)

65 (70.7)

29 (44.6)

$26(44.8) \quad 22(33.8)$

$11(19.0) \quad 12(18.5)$

$6(10.3)$

1 (1.7)

$2(3.1)$

$0(0.0)$

$2(2.0)$

1 (1.1)

$102(100.0)$

$59(57.3)$

43 (42.7)

33 (32.4)

16 (15.7)

28 (27.5)

$8(7.8)$

$5(4.9)$

$6(5.9)$

4 (3.9)

$2(2.0)$

$91(100.0)$

31 (34.1)

21 (23.1)

14 (15.4)

$8(8.8)$

$5(5.5)$

7 (7.7)

4 (4.4)

1 (1.1)

$22(37.3)$

9 (15.3)

$16(27.1)$

3 (5.1)

$2(3.4)$

$3(5.1)$

$2(3.4)$

1 (1.7)

42 (46.2)

$6(14.3)$

$16(38.1)$

7 (16.7)

2 (4.8)

4 (9.5)

$3(7.1)$

$3(7.1)$

1 (2.4)

11 (25.6)

7 (16.3)

12 (27.9)

5 (11.6)

$3(7.0)$

$3(7.0)$

2 (4.7)

1 (2.3)

49 (53.8)

25 (51.0)

5 (10.2)

7 (14.3)

6 (12.2)

$1(2.0)$

$4(8.2)$

$1(2.0)$

$0(0.0)$

$9(9.9)$

1 (2.4)

8 (16.3)

$20(22.0)$

$20(22.0)$

42 (46.2)

$8(19.0)$

$12(24.5)$

$12(24.5)$

18 (36.7)

172 (89.1)

24 (57.1)

83 (90.2)

$1.00(1.00 ; 4.00)$

89 (88.2)

$1.00(1.00 ; 3.00)$

$1.00(1.00 ; 4.00)$

66 (34.2)

43 (22.3)

38 (37.6)

28 (30.4)

26 (25.7)

17 (18.5)

30 (15.5)

12 (11.9)

18 (19.6)

53 (27.5)

23 (43.4)

3 (5.6)

26 (49.1)

$4(7.5)$

$4(7.5)$

41 (40.6)

$17(41.5)$

$3(7.3)$

$21(51.2)$

$4(9.7)$

$3(7.3)$
$12(13.0)$

$6(50.0)$

$0(0.0)$

5 (41.7)

$0(0.0)$

$1(8.3)$ 
Table 2 (continued)

\begin{tabular}{|c|c|c|c|}
\hline \multirow[t]{2}{*}{ Patient characteristics } & \multirow[b]{2}{*}{$\begin{array}{l}\text { Total } \\
N=193(100 \%)\end{array}$} & \multicolumn{2}{|l|}{ MASCC score } \\
\hline & & $\begin{array}{l}<21 \text { (high risk) } \\
N=101(52.3 \%)\end{array}$ & $\begin{array}{l}\geq 21 \text { (low risk) } \\
N=92(47.7 \%)\end{array}$ \\
\hline Fungi identified as source of infection, $n(\%)$ & $4(7.5)$ & $3(7.3)$ & $1(8.3)$ \\
\hline Duration of antibiotics (in days), median (IQR) & $8(6 ; 14)$ & $8(6 ; 15)$ & $8(5 ; 14)$ \\
\hline $\begin{array}{l}\text { Source of infection identified, } n(\%) \\
\text { Pulmonary } \\
\text { Line/port infection } \\
\text { Other } \\
\mathrm{GI}^{\dagger} \\
\mathrm{GU}^{\ddagger} \\
\text { Osteomyelitis } \\
\mathrm{CNS}^{\pi}\end{array}$ & $\begin{array}{l}114(59.1) \\
43(37.7) \\
20(17.5) \\
19(16.7) \\
16(14.0) \\
13(11.4) \\
2(1.8) \\
1(0.9)\end{array}$ & $\begin{array}{l}67(66.3) \\
27(40.3) \\
15(22.4) \\
5(7.5) \\
11(16.4) \\
7(10.4) \\
1(1.5) \\
1(1.5)\end{array}$ & $\begin{array}{l}47(51.1) \\
16(34.0) \\
5(10.6) \\
14(29.8) \\
5(10.6) \\
6(12.8) \\
1(2.1) \\
0(0.0)\end{array}$ \\
\hline $\begin{array}{l}\text { Consults, } n(\%) \\
\text { Hematology/oncology } \\
\text { Infectious diseases } \\
\text { Palliative care }\end{array}$ & $\begin{array}{l}159(82.4) \\
98(50.8) \\
13(6.7)\end{array}$ & $\begin{array}{l}86(85.1) \\
60(59.4) \\
12(11.9)\end{array}$ & $\begin{array}{l}73(79.3) \\
38(41.3) \\
1(1.1)\end{array}$ \\
\hline $\begin{array}{l}\text { Documented goals of care discussion, } n(\%) \\
\text { Primary team } \\
\text { Hematology/oncology team } \\
\text { Primary care physician }\end{array}$ & $\begin{array}{l}24(12.4) \\
19(79.2) \\
4(16.7) \\
1(4.2)\end{array}$ & $\begin{array}{l}21(20.8) \\
16(76.2) \\
4(19.0) \\
1(4.8)\end{array}$ & $\begin{array}{l}3(3.3) \\
3(100.0) \\
0(0.0) \\
0(0.0)\end{array}$ \\
\hline
\end{tabular}

ECOG PS Eastern Cooperative Oncology Group Performance Status, MASCC Multinational Association for Supportive Care in Cancer, GCSF granulocyte colony-stimulating factor, $A M L$ acute myeloid leukemia, $A L L$ acute lymphoblastic leukemia, $C M L$ chronic myeloid leukemia, $C L L$ chronic lymphocytic leukemia, CNS central nervous system, MRSA methicillin-resistant Staphylococcus aureus, IQR interquartile range

${ }^{\alpha}$ As documented in the last oncology note either inpatient or outpatient

${ }^{\eta}$ Other hematological malignancies identified were Myelodysplastic Syndrome, Primary Myelofibrosis, and Waldenström macroglobulinemia

${ }^{\epsilon}$ Includes lung, thymus, mediastinum, trachea, and esophagus

${ }^{\infty}$ Includes pancreas, liver, biliary tract, and gastrointestinal tract (stomach, small intestine, colon, rectum, anal)

${ }^{£}$ Includes bladder, prostate, kidney, testicular, and penile cancer

${ }^{¥}$ Includes ovarian, uterine, vaginal, vulvar, and pelvic masses

${ }^{\beta}$ Fungi found include candida species and Aspergillus species

* Includes pneumonia

${ }^{\Omega}$ Includes all infections that do not fall into any of the other categories. Further information on the type of infection was not collected

${ }^{\dagger}$ Includes colitis and bacterial translocation in the gut

* Includes UTI, cystitis, and pyelonephritis

${ }^{\pi}$ Includes meningitis and spinal epidural abscess

\section{Discussion}

In this single-center retrospective cohort of patients with $\mathrm{FN}$ and cancer (in which more than half of the patients had a hematologic malignancy), nearly $60 \%$ of inpatients had an identifiable source of infection with a substantial number of these testing positive for a virus. MASCC score, although rarely used, was predictive of poor outcomes. Goals of care discussions were rarely initiated, even in patients considered high risk. Our findings suggest a need for hospitalist-focused education efforts and quality improvement initiatives to incorporate MASCC score into oncologic hospitalists' workflow.

The proportion of patients with an identifiable source of infection was higher than prior studies have reported [7, 25].
Most published studies examined patients with fever and neutropenia prior to widespread use of rapid viral testing (e.g., for influenza and RSV). Notably, our study was conducted before the onset of the COVID-19 pandemic, so there were no COVID-positive patients in this sample [26]. The incidence of MRSA and Pseudomonas was low compared to other studies across the USA [7], but most patients received empiric antibiotic therapy appropriately per current guidelines because of the high risk for progression to fatal sepsis and mortality associated with these organisms [7, 9, 10].

Use of MASCC score among inpatients in our study was predictive of poor outcomes including the need for intermediate/ICU level care, respiratory failure necessitating ventilation, septic shock requiring vasopressor use, and 
Table 3 Outcomes

\begin{tabular}{|c|c|c|c|c|c|}
\hline & \multirow{2}{*}{$\begin{array}{l}\text { Total events } \\
N(\%)\end{array}$} & \multicolumn{2}{|l|}{ MASCC score } & \multirow[t]{2}{*}{ AUC } & \multirow{2}{*}{$\begin{array}{l}\text { Absolute risk } \\
\text { Difference }(95 \% \text { CI) }\end{array}$} \\
\hline & & $\begin{array}{l}<21 \text { high risk } \\
101(52.3 \%) \\
N(\%)\end{array}$ & $\begin{array}{l}\geq 21 \text { low risk } \\
92(47.7 \%) \\
N(\%)\end{array}$ & & \\
\hline Inpatient death & $23(11.9)$ & $21(20.8)$ & $2(2.2)$ & 0.721 & $-18.6 \%(-27.1$ to -10.2$)$ \\
\hline Required intermediate or ICU level of care $e^{€}$ & $40(20.7)$ & $37(36.6)$ & $3(3.3)$ & 0.753 & $-33.4 \%(-43.5$ to -23.3$)$ \\
\hline Goals of care discussion & $24(12.4)$ & $21(20.8)$ & $3(3.3)$ & 0.701 & $-17.5 \%(-26.2$ to -8.8$)$ \\
\hline Respiratory failure & $18(9.3)$ & $16(15.8)$ & $2(2.2)$ & 0.702 & $-13.7 \%(-21.4$ to -6.0$)$ \\
\hline Documented delirium & $12(6.2)$ & $11(10.9)$ & $1(1.1)$ & 0.710 & $-9.8 \%(-16.2$ to -3.4$)$ \\
\hline AKI requiring new dialysis ${ }^{\dagger}$ & $2(1.0)$ & $2(2.0)$ & $0(0.0)$ & 0.741 & $-2.0(-4.7$ to 0.7$)$ \\
\hline Septic shock requiring pressors & $19(9.8)$ & $19(18.8)$ & $0(0.0)$ & 0.764 & $-18.8(-26.4$ to -11.2$)$ \\
\hline Positive blood cultures & $53(27.5)$ & $41(40.6)$ & $12(13.0)$ & 0.673 & $-27.6 \%(-39.3$ to -15.8$)$ \\
\hline
\end{tabular}

$A K I$ acute kidney injury, $I C U$ intensive care unit, $A U C$ area under the curve, $C I$ confidence interval

${ }^{\dagger}$ Per the kidney disease: improving global outcomes definition

${ }^{*}$ Requiring use of non-invasive ventilation like continuous positive airway pressure, bi-level continuous positive airway pressure, high-flow nasal cannula; and use of mechanical ventilation with intubation

${ }^{€}$ Intermediate unit defined as a step-up or step-down unit between the general ward and ICU

inpatient death. However, less than $2 \%$ of patients had a MASCC score documented on admission, suggesting that hospitalists do not routinely use this score to identify patients at high risk. MASCC score does have limitations; it was developed as a tool intended to prevent admission or reduce time to discharge for low-risk patients, and it may miss up to $10 \%$ of low-risk patients who later developed complications [15, 19, 27, 28]. However, the Clinical Index of Stable Febrile Neutropenia (CISNE) scaore [29], the alternative to MASCC score, has poor sensitivity and thus has limited usefulness in high-risk patients.

While prior investigators have validated the MASCC score in low-risk patients hospitalized for FN, its use in high-risk patients has been less frequently studied [30-35]. One validation study, with a goal of identifying high-risk patients who might be candidates for hematopoietic growth factor, correctly predicted $98.3 \%$ of low-risk patients and $86.4 \%$ of high-risk patients [30]. Other studies have (a) identified patients at a high risk of complications by calculating the score at 48 and $72 \mathrm{~h}$, adding a measure of organ dysfunction [31]; (b) attempted to combine MASCC score of $<21$ with Creactive protein or procalcitonin to predict poor outcomes [32-34]; and (c) examined MASCC score's calibration to identify the risk of mortality and the need for ICU level of care among patients with hematologic malignancies [35]. These studies were limited in that most were done in the ED setting, and none of them examined the use of goals of care discussions in patients with a high risk of poor outcomes.

It is not clear why goals of care were infrequently discussed with patients. We hypothesize that because neutropenic fever is a common, predictable, and manageable complication of cytotoxic therapy, there is often uncertainty regarding who should initiate these conversations. Internists may believe that goals are best discussed by the primary oncologist treating a patient's cancer. They may also fear that they lack the advanced training required to independently prognosticate various stages of cancer and navigate goals of care in those receiving active systemic therapy, especially in an era of evolving cancer therapeutics. There are also known differences in perception of cancer outcomes between physicians [36], which may have contributed to the failure to initiate conversations. Qualitative studies would help to refine these early hypotheses.

Nearly three-quarters of patients who died during hospitalization in our cohort required intermediate/ICU level of care prior to their death. This suggests that these patients experienced clinical deterioration and, either at that time or prior to it, decided to escalate care. While this may have been consistent with patient preferences, it may also have been a missed opportunity to initiate goals of care conversation between the patient, hospitalists, and oncologists in patients with high-risk FN.

There are limitations to our study. First, it is a single-center study with a small sample size, and these results may not be generalizable to larger populations. Second, this study is a retrospective analysis with a risk of misclassification bias in calculating scores. For example, the MASCC score contains subjective characteristics like "burden of illness" and "dehydration." We did go to great lengths to standardize these definitions across reviewers. However, it is possible that those with worse outcomes were more likely to be classified with a high-risk MASCC score. Validation of the score to identify 
high-risk patients in a larger, multi-institutional sample of inpatients may be warranted. We also did not create multivariable models to examine the association between MASCC score and outcomes. Our goal, however, was primarily to demonstrate that groups with high and low MASCC scores are different, and increased utilization of this scoring system will allow hospitalists to clarify these differences and improve patient care.

\section{Conclusions}

In a single-center study of patients with fever and neutropenia, nearly $60 \%$ had an identifiable source of infection. Although the incidence of multidrug-resistant bacteria was low, patients mostly received empiric antibiotics appropriately to cover for these organisms to reduce the risk of sepsis and mortality per current guidelines. Patients with a high-risk MASCC score had worse outcomes, i.e., higher inpatient mortality and utilization of critical care therapies, but MASCC score was not commonly used by treating clinicians. Routine use of the MASCC score may help clinicians identify patients at high risk for complications, allowing for risk stratification at the time of admission. MASCC score could aid with triage, unit placement, and need to address and document goals of care discussions during hospitalization. Hospitalist-focused education efforts about MASCC score should be considered a way to identify patients at high risk for complications and facilitate goals of care discussions.

Acknowledgment The project utilized a REDCap database funded by grant UL1TR002544, an Institutional Clinical and Translational Service Award (CTSA) from the NIH awarded to Tufts University, School of Medicine.

Author contributions Prarthna V Bhardwaj: Conception and design, Collection and assembly of data, Original Draft, Review and Editing; Megan Emmich: Conception and design, Collection and assembly of data, Original Draft; Fatima Ali: Collection and assembly of data, Original Draft; Ritika Walia: Collection and assembly of data, Original Draft; Prithwijit Roychowdhury: Original Draft; Jackson Clark: Collection and assembly of data, Original Draft; Arthi Sridhar: Collection and assembly of data, Original Draft, Alexander Knee: Data Analysis and interpretation, Review and Editing; Tara Lagu: Conception and design, Review and Editing, Supervision; Kah Poh Loh: Conception and design, Review and Editing, Supervision.

\section{Data Availability Yes}

Code availability N/A

\section{Declarations}

Ethics approval Approved by the IRB at Baystate Medical Center
Consent to participate N/A

\section{Consent for publication N/A}

Conflict of interest Dr. Tara Lagu has no conflict of interest. Dr. Lagu is supported by the National Heart, Lung, and Blood Institute of the National Institutes of Health under Award Numbers R01 HL13998501A1 and 1R01HL146884-01. Dr. Lagu has served as a consultant for the Yale Center for Outcomes Research and Evaluation, under contract to the Centers for Medicare and Medicaid Services, for which she has provided clinical and methodological expertise and input on the development, reevaluation, and implementation of hospital outcome and efficiency measures. The views expressed in this manuscript do not necessarily reflect those of the Yale Center for Outcomes Research and Evaluation or the Centers for Medicare and Medicaid Services.

Dr. Kah Poh Loh is supported by the National Cancer Institute of the National Institute of Health under Award number K99CA237744 and the Wilmot Research Fellowship Award. Dr. Loh also serves as a consultant to Pfizer and Seattle Genetics.

Dr. Prarthna Bhardwaj, Dr. Megan Emmich, Dr. Fatima Ali, Dr. Ritika Walia, Dr. Jackson Clark, Dr. Arthi Sridhar, Mr. Prithwiraj Roychowdhury, and Mr. Alexander Knee do not have any relevant financial or non-financial conflict of interests to disclose.

\section{References}

1. Culakova E, Thota R, Poniewierski MS, Kuderer NM, Wogu AF, Dale DC, Crawford J, Lyman GH (2014) Patterns of chemotherapy-associated toxicity and supportive care in US oncology practice: a nationwide prospective cohort study. Cancer Med 3(2):434-444. https://doi.org/10.1002/cam4.200

2. Flowers CR, Seidenfeld J, Bow EJ, Karten C, Gleason C, Hawley DK, Kuderer NM, Langston AA, Marr KA, Rolston KVI, Ramsey SD (2013) Antimicrobial prophylaxis and outpatient management of fever and neutropenia in adults treated for malignancy: American Society of Clinical Oncology Clinical Practice Guideline. JCO. 31(6):794-810. https://doi.org/10.1200/JCO.2012.45.8661

3. Crawford J, Becker PS, Armitage JO, Blayney DW, Chavez J, Curtin P, Dinner S, Fynan T, Gojo I, Griffiths EA, Hough S, Kloth DD, Kuter DJ, Lyman GH, Mably M, Mukherjee S, Patel S, Perez LE, Poust A, Rampal R, Roy V, Rugo HS, Saad AA, Schwartzberg LS, Shayani S, Talbott M, Vadhan-Raj S, Vasu S, Wadleigh M, Westervelt P, Burns JL, Pluchino L (2017) Myeloid growth factors, Version 2.2017, NCCN Clinical Practice Guidelines in Oncology. J Natl Compr Cancer Netw 15(12): 1520-1541. https://doi.org/10.6004/jnccn.2017.0175

4. Kuderer NM, Dale DC, Crawford J, Cosler LE, Lyman GH (2006) Mortality, morbidity, and cost associated with febrile neutropenia in adult cancer patients. Cancer. 106(10):2258-2266. https://doi.org/ $10.1002 /$ cncr. 21847

5. Al-Tawfiq JA, Hinedi K, Khairallah H et al (2019) Epidemiology and source of infection in patients with febrile neutropenia: a tenyear longitudinal study. J Infect Public Health 12(3):364-366. https://doi.org/10.1016/j.jiph.2018.12.006

6. Lakshmaiah KC, Malabagi AS, Govindbabu, Shetty R, Sinha M, Jayashree RS (2015) Febrile neutropenia in hematological malignancies: clinical and microbiological profile and outcome in high risk patients. J Lab Physicians 7(2):116-120. https://doi.org/10. 4103/0974-2727.163126

7. Freifeld AG, Bow EJ, Sepkowitz KA, Boeckh MJ, Ito JI, Mullen CA, Raad II, Rolston KV, Young JA, Wingard JR, Infectious Diseases Society of America (2011) Clinical practice guideline for the use of antimicrobial agents in neutropenic patients with 
cancer: 2010 Update by the Infectious Diseases Society of America. Clin Infect Dis 52(4):e56-e93. https://doi.org/10.1093/cid/cir073

8. Pizzo PA (1993) Management of fever in patients with cancer and treatment-induced neutropenia. N Engl J Med 328(18):1323-1332. https://doi.org/10.1056/NEJM199305063281808

9. Averbuch D, Orasch C, Cordonnier C, Livermore DM, Mikulska M, Viscoli C, Gyssens IC, Kern WV, Klyasova G, Marchetti O, Engelhard D, Akova M, on behalf of ECIL4, a joint venture of EBMT, EORTC, ICHS, ESGICH/ESCMID and ELN (2013) European guidelines for empirical antibacterial therapy for febrile neutropenic patients in the era of growing resistance: summary of the 2011 4th European Conference on Infections in Leukemia. Haematologica. 98(12):1826-1835. https://doi.org/10.3324/ haematol.2013.091025

10. Schmidt-Hieber M, Teschner D, Maschmeyer G, Schalk E (2019) Management of febrile neutropenia in the perspective of antimicrobial de-escalation and discontinuation. Expert Rev Anti-Infect Ther 17(12):983-995. https://doi.org/10.1080/14787210.2019.1573670

11. Bodey GP, Jadeja L, Elting L (1985) Pseudomonas bacteremia. Retrospective analysis of 410 episodes. Arch Intern Med 145(9): 1621-1629. https://doi.org/10.1001/archinte.145.9.1621

12. Holland T, Fowler VG, Shelburne SA (2014) Invasive grampositive bacterial infection in cancer patients. Clin Infect Dis 59(Suppl 5):S331-S334. https://doi.org/10.1093/cid/ciu598

13. de Naurois J, Novitzky-Basso I, Gill MJ, Marti Marti F, Cullen MH, Roila F (2010) Management of febrile neutropenia: ESMO Clinical Practice Guidelines. Ann Oncol 21:v252-v256. https://doi.org/10. 1093/annonc/mdq196

14. Hughes WT, Armstrong D, Bodey GP, Bow EJ, Brown AE, Calandra T, Feld R, Pizzo PA, Rolston KVI, Shenep JL, Young LS (2002) 2002 Guidelines for the use of antimicrobial agents in neutropenic patients with cancer. Clin Infect Dis 34(6):730-751. https://doi.org/10.1086/339215

15. Klastersky J, Paesmans M, Rubenstein EB, Boyer M, Elting L, Feld R, Gallagher J, Herrstedt J, Rapoport B, Rolston K, Talcott J, for the Study Section on Infections of Multinational Association for Supportive Care in Cancer (2000) The Multinational Association for Supportive Care in Cancer risk index: a multinational scoring system for identifying low-risk febrile neutropenic cancer patients. JCO. 18(16):3038-3051. https://doi.org/10.1200/JCO.2000.18.16. 3038

16. Taj M, Nadeem M, Maqsood S, Shah T, Farzana T, Shamsi TS (2017) Validation of MASCC score for risk stratification in patients of hematological disorders with febrile neutropenia. Indian $\mathrm{J}$ Hematol Blood Transfus 33(3):355-360. https://doi.org/10.1007/ s12288-016-0730-7

17. Gunderson CC, Erickson Britt K, Wilkinson-Ryan I et al (2019) Prospective evaluation of Multinational Association of Supportive Care in Cancer risk index score for gynecologic oncology patients with febrile neutropenia. Am J Clin Oncol 42(2):138-142. https:// doi.org/10.1097/COC.0000000000000498

18. Baskaran ND, Gan GG, Adeeba K (2008) Applying the Multinational Association for Supportive Care in Cancer risk scoring in predicting outcome of febrile neutropenia patients in a cohort of patients. Ann Hematol 87(7):563-569. https://doi.org/10.1007/ s00277-008-0487-7

19. Cherif H, Johansson E, Björkholm M, Kalin M (2006) The feasibility of early hospital discharge with oral antimicrobial therapy in low risk patients with febrile neutropenia following chemotherapy for hematologic malignancies. Haematologica. 91(2):215-222

20. Bitar RA (2015) Utility of the Multinational Association for Supportive Care in Cancer (MASCC) risk index score as a criterion for nonadmission in febrile neutropenic patients with solid tumors. Perm J 19(3):37-47. https://doi.org/10.7812/TPP/14-188

21. Goodman LM, Estfan B, Montero A, Kunapareddy G, Lau J, Gallagher E, Best C, Tripp B, Moeller M, Bolwell B, Stevenson J
(2017) Improving the management of patients with low-risk neutropenic fever at the Cleveland Clinic Taussig Cancer Institute. JOP. 13(3):e259-e265. https://doi.org/10.1200/JOP.2016.017277

22. Aagaard T, Reekie J, Jørgensen M, Roen A, Daugaard G, Specht L, Sengeløv H, Mocroft A, Lundgren J, Helleberg M (2020) Mortality and admission to intensive care units after febrile neutropenia in patients with cancer. Cancer Med. 9(9):3033-3042. https://doi. org/10.1002/cam4.2955

23. Harris PA, Taylor R, Thielke R, Payne J, Gonzalez N, Conde JG (2009) Research electronic data capture (REDCap) - a metadatadriven methodology and workflow process for providing translational research informatics support. J Biomed Inform 42(2):377381. https://doi.org/10.1016/j.jbi.2008.08.010

24. Oken MMMD, Creech RHMD, Tormey DCMD et al (1982) Toxicity and response criteria of the Eastern Cooperative Oncology Group. J Clin Oncol 5(6):649-656

25. Klastersky J, Ameye L, Maertens J, Georgala A, Muanza F, Aoun M, Ferrant A, Rapoport B, Rolston K, Paesmans M (2007) Bacteraemia in febrile neutropenic cancer patients. Int $\mathrm{J}$ Antimicrob Agents 30:51-59. https://doi.org/10.1016/j. ijantimicag.2007.06.012

26. Cooksley T, Font C, Scotte F, Escalante C, Johnson L, Anderson R, Rapoport B Emerging challenges in the evaluation of fever in cancer patients at risk of febrile neutropenia in the era of COVID-19: a MASCC position paper. Support Care Cancer. Published online November 23, 2020:1129-1138. https://doi.org/10.1007/s00520020-05906-y

27. Innes H, Lim SL, Hall A, Chan SY, Bhalla N, Marshall E (2008) Management of febrile neutropenia in solid tumours and lymphomas using the Multinational Association for Supportive Care in Cancer (MASCC) risk index: feasibility and safety in routine clinical practice. Support Care Cancer 16(5):485-491. https://doi.org/ 10.1007/s00520-007-0334-8

28. Klastersky J, Paesmans M, Georgala A, Muanza F, Plehiers B, Dubreucq L, Lalami Y, Aoun M, Barette M (2006) Outpatient oral antibiotics for febrile neutropenic cancer patients using a score predictive for complications. J Clin Oncol 24(25):4129-4134. https:// doi.org/10.1200/JCO.2005.03.9909

29. Carmona-Bayonas A, Gómez J, González-Billalabeitia E, Canteras M, Navarrete A, Gonzálvez ML, Vicente V, Ayala de la Peña F (2011) Prognostic evaluation of febrile neutropenia in apparently stable adult cancer patients. Br J Cancer 105(5):612-617. https:// doi.org/10.1038/bjc.2011.284

30. Uys A, Rapoport BL, Anderson R (2004) Febrile neutropenia: a prospective study to validate the Multinational Association of Supportive Care of Cancer (MASCC) risk-index score. Support Care Cancer 12(8):555-560. https://doi.org/10.1007/s00520-0040614-5

31. Blot F, Nitenberg G (2004) Patients neutropéniques fébriles à bas et haut risques. Presse Med 33(7):467-473. https://doi.org/10.1016/ S0755-4982(04)98634-4

32. Ahn S, Lee Y-S, Chun Y-H, Kwon IH, Kim W, Lim KS, Kim TW, Lee KH (2011) Predictive factors of poor prognosis in cancer patients with chemotherapy-induced febrile neutropenia. Support Care Cancer 19(8):1151-1158. https://doi.org/10.1007/s00520010-0928-4

33. Ahn S, Lee Y-S, Lim KS, Lee J-L (2013) Adding procalcitonin to the MASCC risk-index score could improve risk stratification of patients with febrile neutropenia. Support Care Cancer 21(8):23032308. https://doi.org/10.1007/s00520-013-1787-6

34. Günalp M, Koyunoğlu M, Gürler S et al (2014) Independent factors for prediction of poor outcomes in patients with febrile neutropenia. Med Sci Monit 20:1826-1832. https://doi.org/10.12659/MSM. 892269

35. Combariza JF, Lombana M, Pino LE, Arango M (2015) C-reactive protein and the MASCC risk index identify high-risk patients with 
febrile neutropenia and hematologic neoplasms. Support Care Cancer 23(4):1009-1013. https://doi.org/10.1007/s00520-0142454-2

36. Balanchivadze N, Slota AA, Mangano A, Kudirka A, Alkhatib Y (2020) Physician perceptions about goals of care of hospitalized patients with malignancies. JCO 38(15_suppl):e19234. https://doi. org/10.1200/JCO.2020.38.15 suppl.e19234

37. Klastersky J, Paesmans M, Aoun M, Georgala A, Loizidou A, Lalami Y, Dal Lago L (2016) Clinical research in febrile neutropenia in cancer patients: past achievements and perspectives for the future. World J Clin Infect Dis 6(3):37-60. https://doi.org/ 10.5495/wjcid.v6.i3.37

Publisher's note Springer Nature remains neutral with regard to jurisdictional claims in published maps and institutional affiliations.

\section{Affiliations}

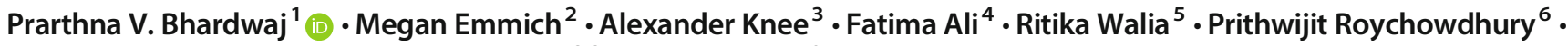 Jackson Clark $^{4} \cdot$ Arthi Sridhar $^{7} \cdot$ Tara Lagu $^{8,9} \cdot$ Kah Poh Loh $^{10}$}

1 Division of Hematology Oncology, University of Massachusetts Medical School-Baystate, 759 Chestnut St, Springfield, MA 01199, USA

2 Division of Hematology Oncology, University of Connecticut, Farmington, CT, USA

3 Office of Research, Epidemiology/Biostatistics Research Core, Baystate Medical Center, Springfield, MA, USA

4 Department of Internal Medicine, University of Massachusetts Medical School-Baystate, Springfield, MA, USA

5 Department of Medicine-Pediatrics, University of Massachusetts Medical School-Baystate, Springfield, MA, USA
6 University of Massachusetts Medical School-Baystate, Springfield, MA, USA

7 Division of Hematology Oncology, University of Texas at Houston, Houston, TX, USA

8 Center for Health Services and Outcomes Research, Institute of Public Health and Medicine, Northwestern Feinberg School of Medicine, Chicago, IL, USA

9 Division of Hospital Medicine, Northwestern Feinberg School of Medicine, Chicago, IL, USA

10 James P Wilmot Cancer Institute, Division of Hematology/ Oncology, Department of Medicine, University of Rochester Medical Center, Rochester, NY, USA 